
STATUS AMONG SOUTH SINAI CHILDREN

doi:10.1136/archdischild-2012-302724.1441

${ }^{1} \mathrm{NE}-\mathrm{M}$ Hassan, ${ }^{1} \mathrm{SA}$ El-Masry, ${ }^{2} \mathrm{GA}$ Yamamah. 'Biological Anthropology; ${ }^{2}$ Peadiatrics, National Research Centre, Cairo, Egypt

Background No information exists on nutritional status of South Sinai residing children, Egypt.

Aim Assessing prevalence of malnutrition among South Sinai children.

Methods Cross sectional study included 3987 healthy children (0 to 11 years); randomly selected; represent about $12 \%$ of all children from the 6 areas of South Sinai. Height and weight were measured. Weight-for-age Z score (WAZ), height-for-age Z score (HAZ) and weight-for-height $Z$ score (HAZ) were used to estimate the children's nutritional status. Venous blood sample was obtained to measure plasma hemoglobin level for school students.

Results Wasting (WHZ<-1.96 SD), underweight (WAZ $<-1.96$ $\mathrm{SD})$ and stunting (HAZ $<-1.96 \mathrm{SD}$ ) were prevalent among $4.2 \%$, $8.9 \%$ and $11 \%$, respectively. Prevalence of underweight, at risk of wasting and be anemic were more prevalent among boys than girls $(p<0.01)$. On the other side, $8 \%$ were overweight and $4 \%$ were obese. Although small percentage of anemic school children was suffering from growth deviation (wasting, 2.8\%; underweight, $5.6 \%$; stunting, $9.9 \%$; overweight, $2.8 \%$ and obese, $1.4 \%$ ), $.55 \%$ of them were at risk of growth deviations (wasting, underweight and stunting). Under nutrition were more prevalent among South Sinai children than their peers in Greater Cairo, while over nutrition was less prevalent.

Conclusion The highest prevalence of malnutrition was detected in infant's age. Anemia of primary school children was more prevalent among those at risk of under nutrition than undernourished ones.

Community education on environmental sanitation and personal hygienic practices, proper child rearing, breast-feeding and weaning practices would possibly reverse the trends.

\section{ELEMENTARY SCHOOL-BASED OBESITY PREVENTION INTERVENTION EFFECT ON WAIST CIRCUMFERENCE AMONG MULTIETHNIC 6-13 YEAR OLDS}

doi:10.1136/archdischild-2012-302724.1442

${ }^{1} \mathrm{D}$ Hollar, ${ }^{2} \mathrm{G}$ Lopez-Mitnik, ${ }^{3} \mathrm{~L}$ Hollar, ${ }^{2} \mathrm{~S}$ Messiah. ${ }^{1}$ Mississippi Food Network/University of Miami Miller School of Medicine; 'University of Miami Miller School of Medicine, Miami; ${ }^{3}$ Nova Southeastern University College of Osteopathic Medicine, Fort Lauderdale, FL, USA

Background Childhood onset obesity and related health consequences continue to be major clinical and public health issues in the USA and abroad. Schools provide an opportunity to implement prevention strategies to large, diverse pediatric audiences. Healthier Options for Public Schoolchildren (HOPS) was a school-based obesity prevention intervention with nutrition and physical activity components implemented in the elementary school setting targeting 6-13 year olds.

Methods HOPS was a quasi-experimental elementary schoolbased obesity prevention intervention targeting ethnically diverse 6-13-year-olds (Kindergarten-6 ${ }^{\text {th }}$ ). Over four school years (August 2004-June 2009), five schools (four intervention; one control, $\mathrm{N}=3,183,48 \%$ Hispanic) in Florida participated in the study. Waist circumference (WC) data was reported in the Fall of 2005 and Spring of 2006 only and these one year results are reported here.

Results Among boys, the mean incremental change in WC (measured in centimeters $[\mathrm{cm}]$ ) increase was significantly less in the intervention $(1.35 \mathrm{~cm}+/-0.88$ [SD]) versus control schools $(3.83 \mathrm{~cm}$ $+/-0.94)(\mathrm{P}<0.0001)$. Among girls the mean incremental change in
WC increase was significantly less in the intervention $(1.20 \mathrm{~cm}+/-$ $0.84)$ versus control schools $(4.17 \mathrm{~cm}+/-0.89)(\mathrm{P}<0.0001)$. Similarly, waist-to-height ratio results showed that the intervention group mean incremental change was significantly less versus the control group for boys $(\mathrm{P}=0.0002)$ and girls $(\mathrm{P}<0.0001)$.

Conclusions Elevated WC is strongly correlated with cardiometabolic disease risk factors and should be monitored in young children as such. School-based obesity prevention interventions show promise in improving weight and potentially cardiometabolic health in elementary-school aged children.

\section{RELATION OF BIRTH WEIGHT AND INFANT GROWTH WITH BODY SIZE, BODY COMPOSITION AND FAT DISTRIBUTION AT AGE 5-6 YEARS}

doi:10.1136/archdischild-2012-302724.1443

${ }^{1} \mathrm{M}$ De Beer, ${ }^{2} \mathrm{M}$ Van Eijsden, ${ }^{3} \mathrm{~T}$ Vrijkotte, ${ }^{4} \mathrm{C}$ Fall, ${ }^{4} \mathrm{C}$ Osmond, ${ }^{1} \mathrm{R}$ Gemke. ${ }^{1}$ Pediatrics, VU University Medical Center; ${ }^{2} E$ Eidemiology, Documentation and Health Promotion, Municipal Health Service; ${ }^{3}$ Social Medicine, Academic Medical Center, Amsterdam, The Netherlands; ${ }^{4}$ MRC Lifecourse Epidemiology Unit, Southampton General Hospital, Southampton, UK

Background and Aims In a prospective cohort study (the $A B C D$ study), we investigated the association of early growth with (precursors of) childhood obesity.

Methods Our study was based on 2,338 term born children with on average 7 measures of growth (weight and length) from birth to age 12 months. We used conditional weight, a residual of current weight regressed on prior weights, to represent deviations from expected weight gain from 0 to 1,1 to 3,3 to 6 , and 6 to 12 months. The same method was applied for conditional length and body mass index. Relations of these measures with height, body mass index, fat mass (adjusted for height and fat free mass) and waist-to-height ratio (WHtR) at age 5-6 were explored using linear regression analysis.

Results Higher birth weight, weigh conditionals and bmi conditionals for all periods in infancy (expressing faster growth) were associated with increased height, body mass index, fat mass and WHtR. Furthermore, although higher length conditionals after 3 months were associated with higher childhood bmi, no association was found with fat mass. Higher length conditionals were associated with lower WHtR.

Conclusions Faster prenatal growth and subsequent faster infant weight- and bmi gain were associated with greater childhood body size, more fat tissue mass and more central adipose distribution. Conversely, although rapid length gain after 3 months was associated with greater childhood body size, this was not accompanied by more fat tissue mass, yet with less central adipose distribution.

\section{ASSESSMENT OF ALIMENTARY BEHAVIOR AND PHYSICAL ACTIVITY IN ROMANIAN OBESE SCHOOL CHILDREN}

doi:10.1136/archdischild-2012-302724.1444

SC Cosoveanu, IO Petrescu, I Puiu. 2nd Pediatric Clinic, Emergency County Hospital, University of Medicine and Pharmacy Craiova, Craiova, Romania

Background and Aims Obesity represents a major, global health problem, continuously increasing all over the world, including Romania. We carried out an epidemiological study on the alimentary behavior and physical activity in overweight and obese school children.

Methods The target population was represented by 41 overweight school children, 68 obese and 57 normal weight, from Craiova, in 2008-2011. Inclusion criteria: children aged 6-14 years with BMI $\geq 95$ percentile/sex/age for obesity, $85 \leq \mathrm{BMI}<95$ percentile/sex/age for overweight and $85<\mathrm{IMC} \leq 5$ percentile/sex/age for normal weight. For every child in our group we followed the alimentary inquiry, the 
physical activity inquiry, the connection between physical activity and food intake.

Results The overweight prevalence was $7.1 \%$ and that of obesity $12.2 \%$. The overweight and obese children, as compared to the normal weight ones eat, daily, sweets $(57.7 \%, 62.1 \%$ vs. $12.3 \%)$, soft drinks $(23.9 \%, 39.9 \%$ vs. $5.2 \%)$, fast-food $(22.8 \%$, $36.7 \%$ vs. $9.4 \%)$, meat products $(54.6 \%, 76.1 \%$ vs. $43.8 \%)$, fruits $(16.8 \%, 7.3 \%$ vs. $48.5 \%)$, vegetables $(41.3 \%, 13.9 \%$ vs. $84.5 \%)$, cereals $(31.1 \%, 24.7 \%$ vs. $49.8 \%$ ), milk products $(18.1 \%, 32.2 \%$ vs. $62.3 \%) .54 .7 \%$ of the overweight children and $60.1 \%$ of the obese do not perform any physical activity in their spare time, as compared to the normal weight $(29.8 \%)$. The food consumption while watching TV or playing on the computer was associated with overweight and obesity in children.

Conclusions Following the nutritional and physical activity investigation in the school children, I noticed an alimentary abuse, both quantitative and qualitative, associated with a physical activity decrease.

\section{THE GROWTH AND NUTRITIONAL STATUS AMONG SCHOOL CHILDREN IN NOVI SAD IN SCHOOL YEAR 2010/2011}

doi:10.1136/archdischild-2012-302724.1445

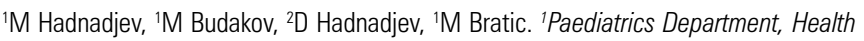
Centre Novi Sad; ${ }^{2}$ Center for Radiology, Clinical Center Vojvodina, Novi Sad, Serbia

Aim To examine physical growth and nutritional status of school children in Novi Sad, during the school year 2010/2011 and determine differences in relation to primary and secondary school children.

Methods On the medical examination of school children aged 7-14 (primary school) and 15-19 (secondary school) in Novi Sad we registered the total number of children: with normal weight (from $-1.5 \mathrm{SD}$ to $+1.5 \mathrm{SD}$ ), obese (+1.5 to $3 \mathrm{SD}$ ) and thin children (from$1.5 \mathrm{SD}$ to $-3 \mathrm{SD}$ ); of average height (from $-1.5 \mathrm{SD}$ to+1.5SD), tall $(+2 \mathrm{SD},+3 \mathrm{SD})$ and short $(-2 \mathrm{SD},-3 \mathrm{SD})$ (nomogram provided by Vukovic).

Results From the total number of the enrolled pupils(n=40861) we have examined $37.25 \%(\mathrm{n}=15222)$ : there were $12783(83.98 \%)$ of average height, $2325(15.27 \%)$ tall pupils and low height pupils 114 $(0.75 \%) ; 11990(78.77 \%)$ of well-nourished, obese $2404(15.79 \%)$ and underweight children 828 (5.44\%).After comparing the data from primary and secondary schools, a statistically significant difference, considering a total number of tall, short, obese, and thin pupils, was in favor of primary school children $(p<0.01)$. There were more children with normal weight in secondary schools $(p>0.05)$, and more obese children in primary schools ( $p>0.05)$.

Conclusion During 2010/2011, the assessment of growth and nutritional status results of school children were in favor of primary school children. There were more children with normal weight children in secondary schools, and more obese children in primary schools, but the differences were not significant.

\section{INFANT FEEDING CHOICES MAY HAVE GENDER EFFECT ON GROWTH DURING INFANCY}

doi:10.1136/archdischild-2012-302724.1446

K Nagahara, K Dobashi, K Itabashi. Pediatrics, Showa University, Tokyo, Japan

Background and Aims A retrospective analysis of healthy Japanese children was conducted to determine whether the growth pattern is altered by breast-feeding (BF) or formula-feeding (FF).

Methods The data of 204 elementary students, age 6 to 9 years were obtained from their parents by questionnaires. The BF and FF group were defined as those fed by only breast milk or formula at 4 months of age. Seventy-one children (31 boys, 40 girls) were in BF and 30 (19 boys, 11 girls) were in FF. The anthropometric data at birth, 1, 4, 7, 10, 18, 36 months-old and investigation time in BF was compared to those in FF.

Result No significant differences were observed in the anthropometric data between $\mathrm{BF}$ and FF girls. The BF boys mean body weight (BW) at 18 months was almost $1,000 \mathrm{~g}$ lower than those in FF. The BW-SD score was significantly smaller from 4 to18 months, and BMI was lower from 10 to 36 months in BF boys. However, both BWSD and BMI had no differences between BF and FF boys from 6 to 9 years. Multiple regression analyses showed that the birth weight, prepregnancy BW, and infant feeding choice were significant factors associated with BWSD, and feeding choice was the only significant factor associated with BMI at 18 and 36 months.

Conclusion Infant feeding choices may have gender effect on growth during infancy. When we evaluate infant growth, we should consider not only infant feeding choices, but also gender, birth weight and prepregnancy BW.

\section{WEIGHTING THE FACTORS ASSOCIATED WITH CHILDREN OBESITY: AN INTERNATIONAL PERSPECTIVE TOWARD AN UNIFIED MODEL}

doi:10.1136/archdischild-2012-302724.1447

D Gregori, I Baldi, OBEY-AD Study Group. Cardiologic, Thoracic and Vascular Sciences, University of Padova, Padova, Italy

Beside genetic predisposition, several factors have been proposed to promote overweight and eventually obesity in children, from the socio- and built- environment down to behavioural attitudes (Bouchard, 2007). How this model is shared by different cultural settings is however unclear and less investigated.

Using a unified protocol for data collection, a cross-sectional study has been performed on 960 children in India, Italy, Germany, France, UK, Argentina, Mexico and Brazil. Children CDC z-scores for BMI have been evaluated in association with several known factors influencing overweight and obesity (maternal and neonatal aspects, socio-economic familiar status, BMI of parents, physical activity, nutrition habits, screen activities). Based on a randomeffect mixed effect model and the Kullback-Leibler Entropy Measure, the capability to explain variability in BMI of such factors has been computed. Percentages of explained variation are given as follows: India $41.4 \%$, Italy $46.6 \%$, Germany $65.6 \%$, France $52.3 \%$, UK $70.1 \%$, Argentina $62.3 \%$, Brazil $59.7 \%$, Mexico $58.2 \%$. Capability of proposed factors to capture variability in BMI is significantly higher in UK ( $p=0.03$ ) than in other countries, being significantly lower in an emerging country like India $(p=0.042)$. These results may suggest that more intense research should be specifically targeted to capture risk factors which are specific for that given cultural setting in addition to the general ones.

\begin{tabular}{l}
\hline 1448 EARLY FORMULA FEEDING PRACTICES AND THEIR \\
POTENTIAL CONTRIBUTION TO LATER OBESITY RISK
\end{tabular}

doi:10.1136/archdischild-2012-302724.1448

${ }^{1} \mathrm{R}$ Tarrant, ${ }^{2} \mathrm{M}$ Sheridan-Pereira, ${ }^{3} \mathrm{~K}$ Younger, ${ }^{3 \mathrm{~J}}$ Kearney. ${ }^{1}$ Department of Clinical Nutrition and Dietetics, Our Lady's Children's Hospital; 'Department of Paediatrics and Newborn Medicine, Coombe Women and Infants University Hospital; ${ }^{3}$ Department of Biological Sciences, Dublin Institute of Technology, Dublin, Ireland

Background and Aims Early feeding practices, including early introduction to solid foods and overfeeding, are known risk factors for childhood obesity. This study aimed to assess maternal formula feeding practices and infant formula feeding patterns, factors that are known to potentially contribute to later obesity risk.

Methods This Irish prospective observational study involved the recruitment and follow-up of 450 eligible mother-infant pairs to 6 weeks postpartum. Data related to formula milk consumption patterns, formula type/brand changing, additions of solids to bottle 